\title{
Resistance to irradiation of micro-algae growing in the storage pools of a nuclear reactor investigated by NMR and neutron spectroscopies
}

\author{
Corinne Rivasseau ${ }^{\mathrm{a}, *}$, Emmanuel Farhi ${ }^{\mathrm{b}}$, Marina Gromova ${ }^{\mathrm{c}}$, Jacques Ollivier ${ }^{\mathrm{b}}$ and \\ Richard Bligny ${ }^{\text {a }}$ \\ ${ }^{a}$ CEA, DSV, iRTSV, Laboratoire de Physiologie Cellulaire Végétale, CNRS, Grenoble, France \\ ${ }^{\mathrm{b}}$ Institut Laue Langevin, Grenoble, France \\ ${ }^{\mathrm{c}}$ CEA, DSM, INAC, SCIB, Grenoble, France
}

\begin{abstract}
A green Chlorophycean micro-alga surviving high irradiation doses has been discovered in the storage pools of a nuclear reactor. Investigating the mechanisms sustaining its properties is of peculiar interest. Metabolic impact of irradiation on the micro-alga as well as structural macromolecular impact were investigated using nuclear magnetic resonance and neutron backscattering spectroscopy. Carbohydrate, amino acid and organic acid content studied as a function of the irradiation dose revealed an intense protein repair activity. Resilience was extracted according to irradiation dose and compared to that of other extremophile species.
\end{abstract}

Keywords: Nuclear magnetic resonance, neutron backscattering spectroscopy, radioresistance, micro-alga, Chlorophyceae

\section{Introduction}

Life develops everywhere on earth including extreme environments. Species surviving high salt concentrations, toxic metals, high pressures, acidity, low or high temperatures [5,6], vacuum and desiccation have been reported. Additionally, some bacteria, among which the champion Deinococcus radiodurans, exhibit an extraordinary ability to survive ionizing irradiation, up to $15 \mathrm{kGy}$. We discovered an ionizing-irradiation tolerant micro-alga growing in the light water cooling systems and in the nuclear waste fuel elements storage pool of the Institut Laue Langevin (ILL) research reactor. This unicellular green micro-alga belonging to the Chlorophyceae class lives just a few meters away from the core in a highly radio-active environment and in a very poor nutrient medium. It is the only eukaryotic autotrophic organism to grow in such environment, for at least 15 years, although contamination possibilities from outside environment are not unusual. This adaptation indicates an irradiation tolerance capacity higher

\footnotetext{
${ }^{*}$ Corresponding author: Corinne Rivasseau, CEA, DSV, iRTSV, Laboratoire de Physiologie Cellulaire Végétale, UMR5168, CNRS, 17 rue des Martyrs, 38054 Grenoble Cedex 9, France. Tel.: +33 4387896 66; Fax: +33 43878 50 91; E-mail: corinne.rivasseau@cea.fr.
} 
than that of other eukaryotes, and a probable adaptation to this environment. There is little knowledge on algae resistance to gamma irradiation and the ability of this micro-alga to tolerate ionizing irradiation has never been reported. Its study is consequently of peculiar interest, all the more as it concerns a eukaryotic organism which is much more complex and fragile than a bacteria. Understanding mechanisms involved in resistance to irradiation in this organism might bring new insights into radiation response of other eukaryotic organisms including animals. We investigated the effects of irradiation on the micro-alga physiology. Micro-algae were stressed with irradiation doses up to $20 \mathrm{kGy}$ ( $2 \mathrm{Mrad}$ ). Their survival was assessed as a function of the irradiation dose. It was found that this Chlorophyceae green micro-alga resists high $\gamma$-irradiation doses, up to $6 \mathrm{kGy}$. Higher doses induce a sensible decrease in the survival rate, but the micro-algae colonies may still recover after a month as shown by the recovery of the physiological parameter photosynthesis. Moreover, after being exposed to up to $20 \mathrm{kGy}$, these algae re-colonize their culture medium within some weeks [2]. Even though the associated tolerance mechanism is not known today, we performed spectroscopic investigations which provided a few macroscopic indicators about the cell behaviour during irradiation. Changes in metabolism and internal structure brought by irradiation were studied respectively using nuclear magnetic resonance $\left({ }^{1} \mathrm{H} \mathrm{NMR}\right)$ and neutron backscattering on the IN13 instrument at the ILL (elastic incoherent backscattering as a function of temperature).

\section{Materials and methods}

\subsection{Nuclear magnetic resonance}

Prior to NMR analysis, hydrophilic metabolites were extracted from control and irradiated samples in an acidic medium, internal standard (maleate) was added, $\mathrm{pH}$ was adjusted to 7.00, (trimethyl)propionate-2,2,3,3- $\mathrm{D}_{4}$ was added, and samples were dissolved in $\mathrm{D}_{2} \mathrm{O}$ as described in [2]. ${ }^{1} \mathrm{H}$ NMR spectra were recorded at $25^{\circ} \mathrm{C}$ using a $400 \mathrm{MHz}$ Bruker (Wissembourg, France) spectrometer equipped with a $5 \mathrm{~mm}$ direct probe with following parameters: free induction decay resolution $0.13 \mathrm{~Hz}$, pulse angle $60^{\circ}$, repetition period $3.82 \mathrm{~s}, 512$ transients. Free induction decays were Fourier transformed with $0.3 \mathrm{~Hz}$ line broadening, phased, baseline corrected and aligned by shifting (trimethyl)propionate-2,2,3,3- $\mathrm{D}_{4}$ signal to zero. Metabolites were identified by their chemical shift and by spiking. For quantification, peak areas were integrated using the WIN-NMR software (Bruker Biospin, Karlsruhe, Germany).

\subsection{Neutron backscattering}

The high resolution thermal backscattering neutron spectrometer IN13 at the ILL was used with an incoming beam wavelength of $2.23 \AA$. The measurement range corresponds to length scales within $1.2<r<21 \AA$ (i.e., include the size of protein side chains and amino acids) and times within $6<t<$ $700 \mathrm{ps}$. Samples of about $1.1 \mathrm{~g}$ were washed with heavy water to remove intercellular contribution and inserted inside aluminium cells, resulting in a beam transmission of $90 \%$. The neutron scattering signal is recorded for each irradiated sample as a function of temperature from 280 up to $310 \mathrm{~K}$. Following Bicout and Zaccai [1] the integrated incoherent elastic signal, corrected from background and empty cell contributions, provides an estimate of the movement of intracellular macromolecules in the instrument spatial and time window. More specifically, if we denote $\left\langle u^{2}\right\rangle$ as the mean square displacement of these molecules, it can be shown that the scattering signal is expressed as $I_{i n c}(q, \omega=0)=I_{0} \exp \left[-q^{2}\left\langle u^{2}\right\rangle / 6\right]$ 
where $q$ and $\omega$ are the neutron momentum and energy transfer in the cell, and $I_{0}$ is a constant, under the condition $\left\langle u^{2}\right\rangle q^{2} \approx 2$. The slope of $\ln \left(I_{\text {inc }}\right)$ as a function of $q^{2}$ in the range $1.2<q<2.2 \AA^{-1}$ gives an estimate of $\left\langle u^{2}\right\rangle$ in the time and space ranges measurable by the instrument, for each temperature and irradiation dose. The resilience spring force constant of macromolecules is then obtained from the relation $k=0.00276 /\left(\mathrm{d}\left\langle u^{2}\right\rangle / \mathrm{d} T\right)[1,5]$.

\section{Results and discussion}

Effects of irradiation on the micro-alga metabolism and structural changes were investigated. The metabolic content defines the biochemical status of the algal cell and its determination provides information on cellular functioning and pathways affected by irradiation. ${ }^{1} \mathrm{H}$ NMR is a sensitive spectroscopic technique enabling the simultaneous identification and quantification of a large number of metabolites contained in the micro-alga (Fig. 1A). Concentration of the main carbohydrates, namely saccharose, trehalose and glucose, amino-acids (glutamate, alanine, valine, leucine and isoleucine) and organic acids (malate and succinate) are reported as a function of irradiation in Table 1. Under irradiation (450-2000 Gy), carbohydrate concentration remains high, indicating that the sugar energy source pathways are affected but still maintained. Furthermore, the pools of some free amino acids increased in the cell, even at low irradiation doses that do not result in cell mortality (450 Gy). This concerned particularly the most hydrophobic amino acids constituting cell membranes such as valine, leucine and isoleucine. Last, at very high radiation doses $(20 \mathrm{kGy})$, most molecules disappeared as most metabolic pathways stopped functioning. Irradiation induces highly excited electronic states in water and macromolecules such as DNA and proteins, producing free radicals like reactive oxygen species, breaking proteins, causing DNA single- and double-strand breaks, and generating damaged bases. Increase in free amino acids content may directly result from protein degradation. It may also be related to damaged
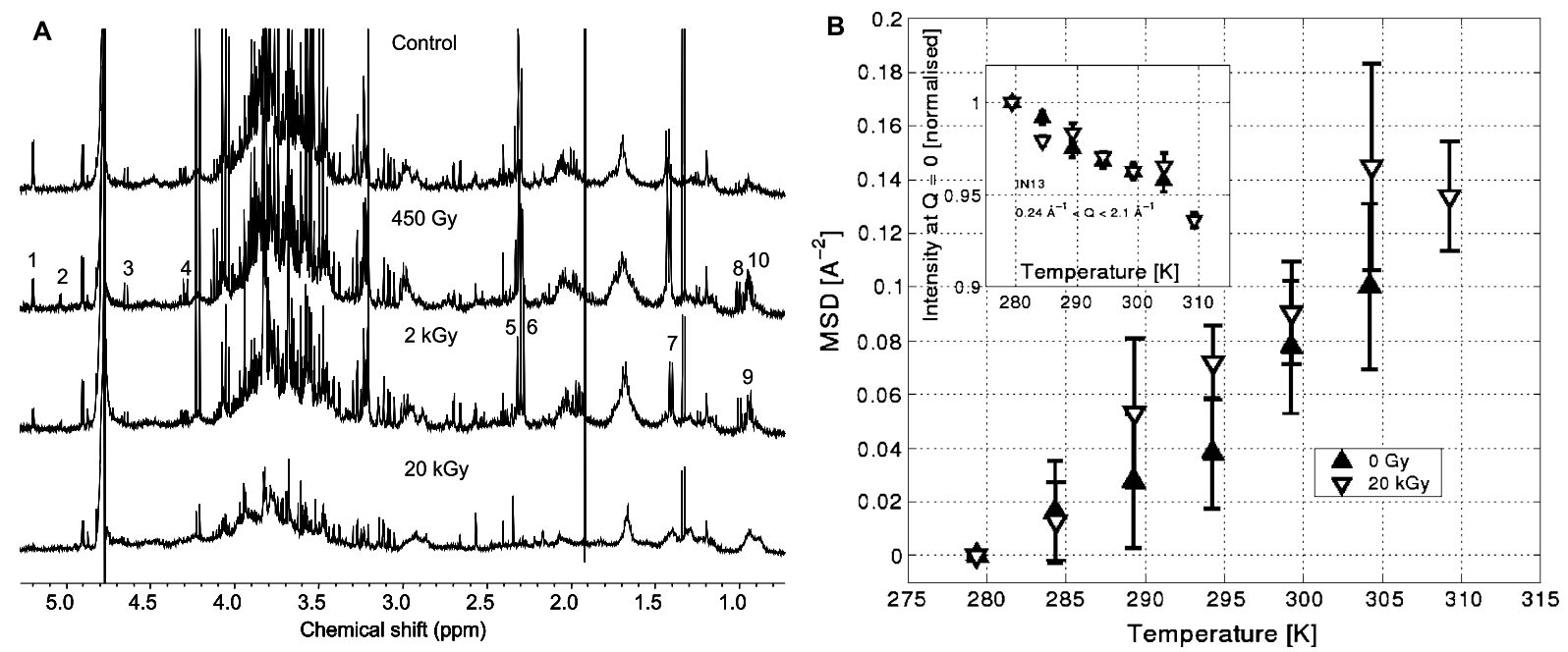

Fig. 1. (A) Micro-alga metabolic profile measured using ${ }^{1} \mathrm{H}$ NMR and (B) mean square displacement measured using neutron backscattering as a function of the irradiation dose. Resilience was extracted from the slope. Insert shows integrated intensity versus temperature. (1) saccharose; (2) trehalose; (3) glucose; (4) malate; (5) succinate; (6) glutamate; (7) alanine; (8) valine; (9) leucine; (10) isoleucine. 
Table 1

Effect of irradiation on metabolic content (metabolite concentration, in $\mu \mathrm{mol} / \mathrm{g}$ wet weight)

\begin{tabular}{lcccc}
\hline & \multicolumn{4}{c}{ Irradiation dose (Gy) } \\
\cline { 2 - 5 } & 0 & 450 & 2000 & 20,000 \\
\hline Sucrose & 3.07 & 2.91 & 1.70 & 0.26 \\
Trehalose & 0.60 & 0.43 & 0.32 & 0.04 \\
Glucose & 0.27 & 0.23 & 0.18 & 0.08 \\
Malate & 0.23 & 0.16 & 0.20 & 0.01 \\
Succinate & 0.05 & 0.04 & 0.03 & 0.02 \\
Glutamate & 2.49 & 2.13 & 2.40 & 0.12 \\
Alanine & 0.57 & 0.87 & 0.46 & 0.00 \\
Valine & 0.05 & 0.10 & 0.08 & 0.01 \\
Leucine & 0.04 & 0.08 & 0.07 & 0.00 \\
Isoleucine & 0.03 & 0.07 & 0.04 & 0.01 \\
\hline
\end{tabular}

DNA repair mechanisms which require amino acids synthesis. But it is also mainly and most probably related to an intense protein repair activity as suggested by the sustained sugar concentration. Repair mechanisms actually require consequent energy. Mitochondrial consumption of carbohydrates, usually used as cell energy source, increases and results in the depletion of the cytosolic carbohydrate pool. The important vacuolar carbohydrate stock will then be released to the cytosol but very slowly, as well as hydrolyzed starch from the plastids. As a result, global cell carbohydrate content remains high while mitochondria that produce energy through carbohydrate oxidation are starving. This starvation triggers macro-autophagy reactions to provide respiration with substrate [3]. Autophagy of membrane proteins or of already broken protein fragments will result in the observed amino acid increase.

From the elastic neutron backscattering signal, the mean square displacement could be extracted as a function of the micro-alga temperature and the irradiation dose. The control resilience value of this micro-alga species adapted to an irradiative environment compares to that previously measured on IN13 for extremophile bacteria living at 75 and $85^{\circ} \mathrm{C}$ which were found as $0.67 \pm 0.11$ and $0.60 \pm 0.01 \mathrm{~N} / \mathrm{m}$, respectively [4]. Even though the error bars indicated for each data point in Fig. 1B are significant, a linear fit using the whole data set reveals a finite change of slope $\mathrm{d}\left\langle u^{2}\right\rangle / \mathrm{d} T$ between the control and the $20 \mathrm{kGy}$ irradiated samples, resulting in a small variation of the associated force constant $k$ (resilience) [1] with values of $0.54 \pm 0.05$ and $0.69 \pm 0.11 \mathrm{~N} / \mathrm{m}$, respectively. This limited resilience increase with additional irradiation may indicate a decrease of the flexibility of the micro-alga macromolecules measured by the IN13 spectrometer. This suggest that the neutralization of major biochemical pathways with irradiation possibly tends to reduce the mean conformational cage extension and increases the stiffness of cellular molecules in their environment. However, the effect of irradiation is mainly on the activity of the organism and the related chemical composition, while limited effects are detected on the picoseconds time-scale dynamics revealed by neutron scattering.

Whereas most neutron backscattering biological studies are performed with crystalline or monodisperse samples, this work addresses living biological material. A homogeneous algal population possessing stable physiological characteristics was analyzed. Original combination of complementary spectroscopic techniques gave new insight in understanding the behaviour of this complex eukaryotic organism under irradiation. 


\section{References}

[1] D.J. Bicout and G. Zaccai, Biophys. J. 80 (2001), 1115-1123.

[2] E. Farhi, C. Rivasseau, M. Gromova, E. Compagnon, V. Marzloff, J. Ollivier, A.M. Boisson, R. Bligny, F. Natali, D. Russo and A. Couté, J. Phys. Condens. Matter. 20 (2008), 104216.

[3] P. Genix, R. Bligny, J.-P. Martin and R. Douce, Plant. Physiol. 94 (1990), 717-722.

[4] M. Tehei, B. Franzetti, D. Madern, M. Ginzburg, B.-Z. Ginzburg, M.-T. Guidici-Orticoni, M. Bruschi and G. Zaccai, Eur. Mol. Biol. Org. Rep. 5 (2004), 66-70.

[5] M. Tehei, B. Franzetti, K. Wood, F. Gabel, E. Fabiani, M. Jasnin, M. Zamponi, D. Oesterhelt, G. Zaccai, M. Ginzburg and B.-Z. Ginzburg, Proc. Natl. Acad. Sci. USA 104 (2007), 766-771.

[6] M. Tehei and G. Zaccai, Biochim. Biophys. Acta 1724 (2005), 404. 


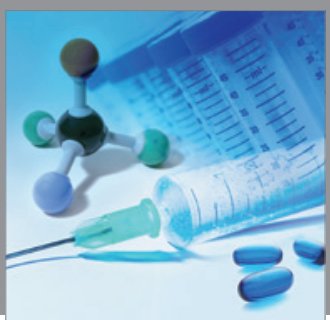

International Journal of

Medicinal Chemistry

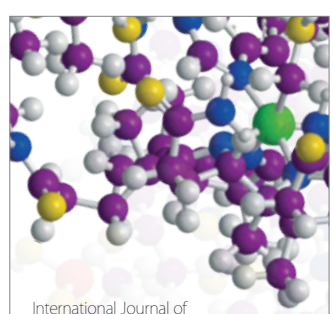

Carbohydrate Chemistry

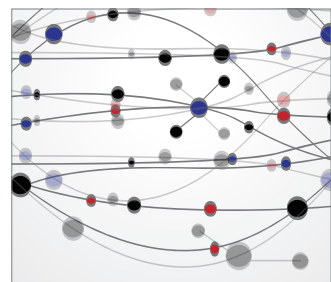

The Scientific World Journal
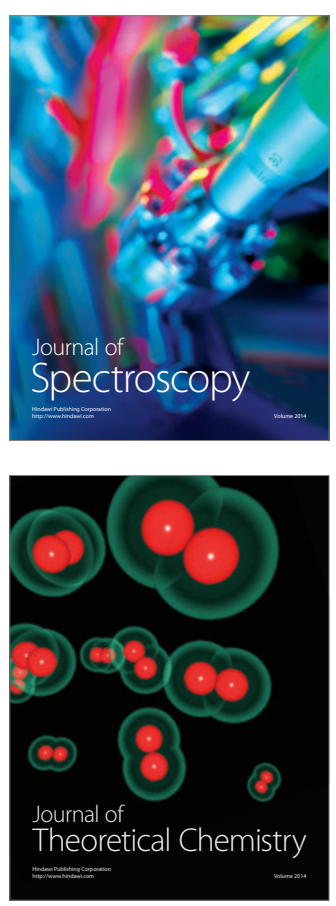
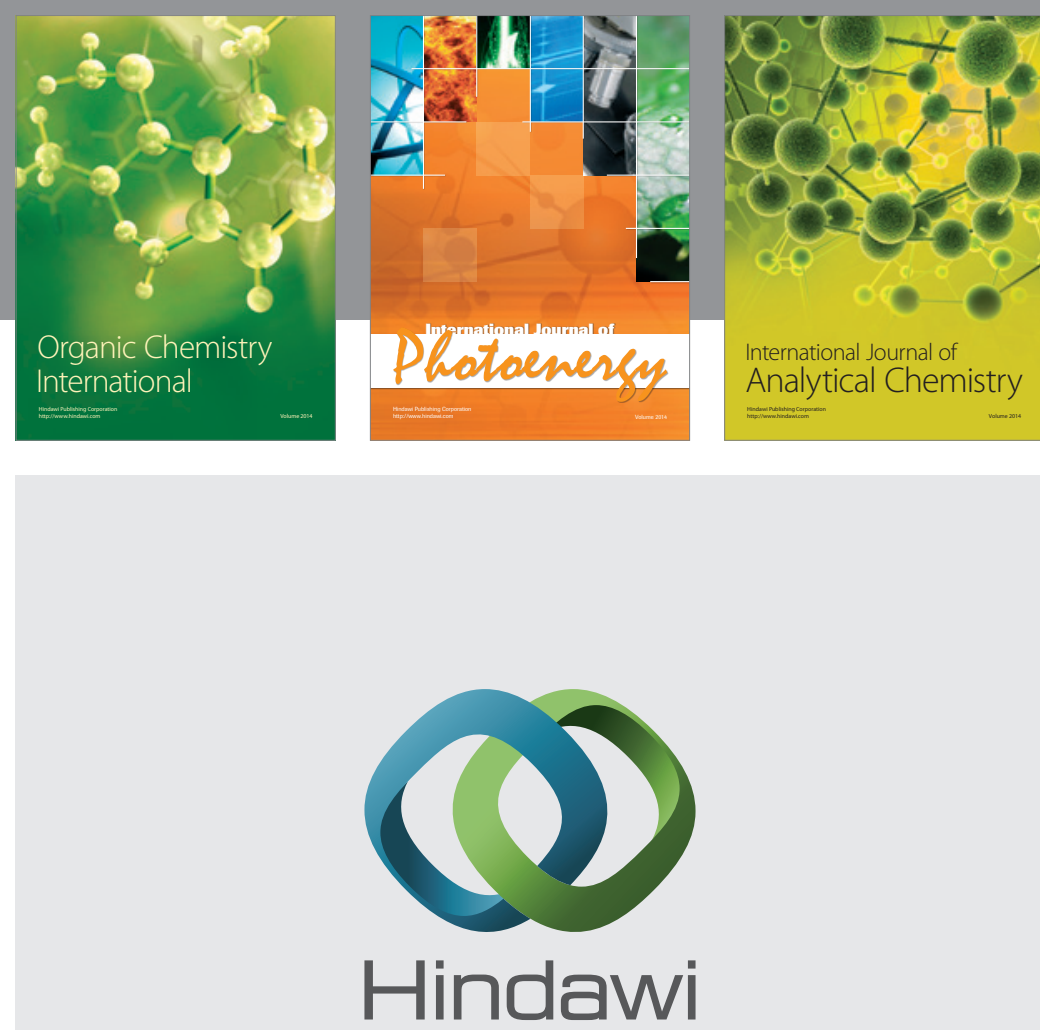

Submit your manuscripts at

http://www.hindawi.com
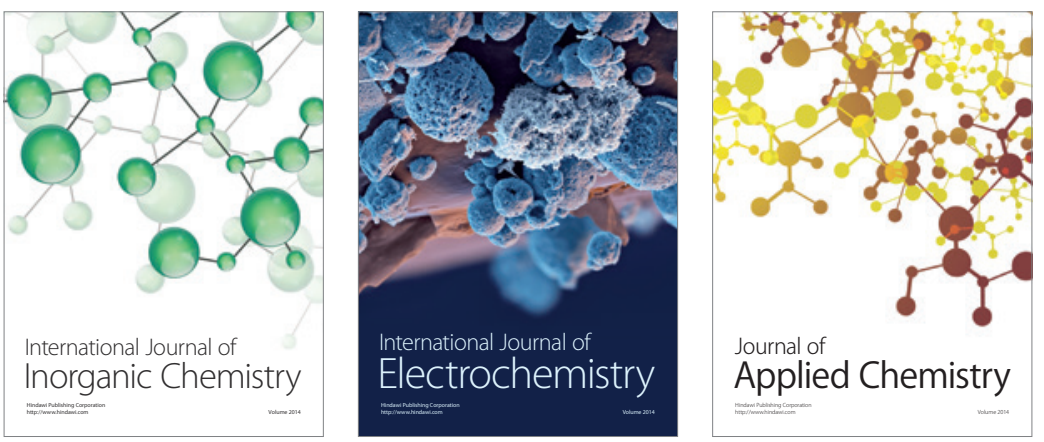

Journal of

Applied Chemistry
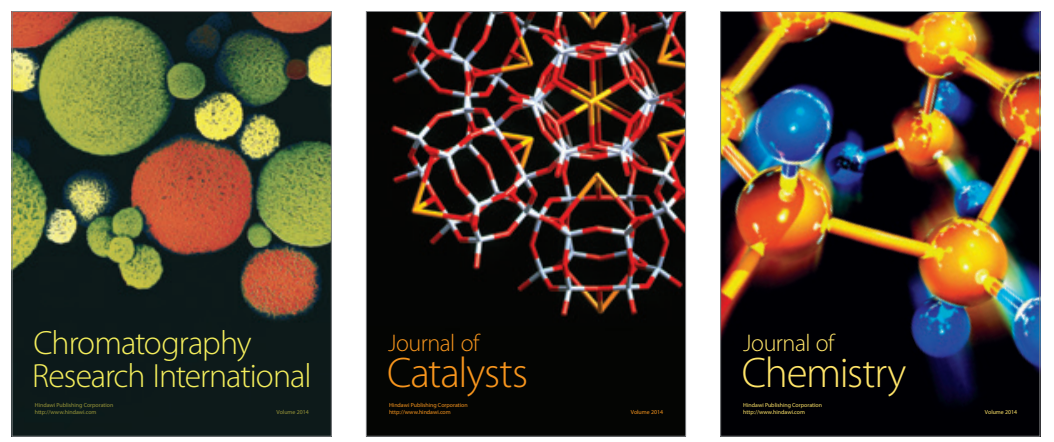
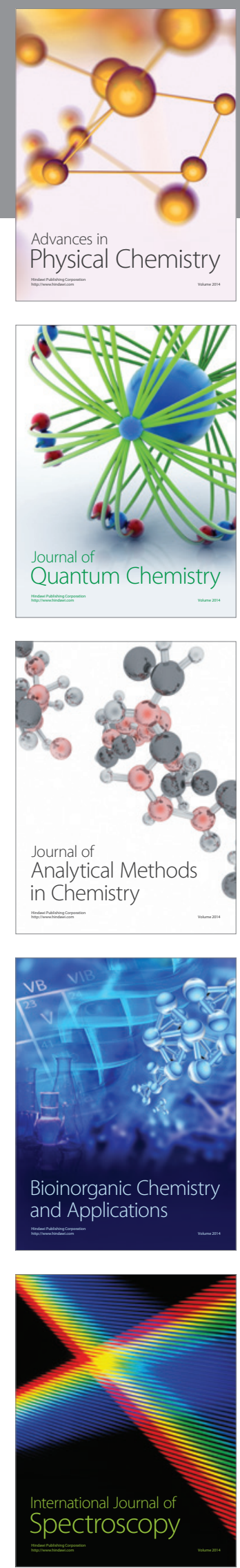